\title{
Determination of the number of light neutrino species
}

\section{L3 Collaboration}

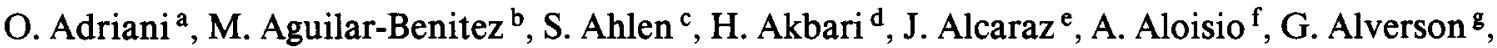
M.G. Alviggi ${ }^{\mathrm{f}}$, G. Ambrosi ${ }^{\mathrm{h}}$, Q. An ${ }^{\mathrm{i}}$, H. Anderhub ${ }^{\mathrm{j}}$, A.L. Anderson ${ }^{\mathrm{k}}$, V.P. Andreev ${ }^{\ell}$, T. Angelov ${ }^{\mathrm{k}}$, L. Antonov ${ }^{\mathrm{m}}$, D. Antreasyan ${ }^{\mathrm{n}}, \mathrm{P}$. Arce ${ }^{\mathrm{b}}$, A. Arefiev ${ }^{\mathrm{o}}$, A. Atamanchuk ${ }^{\ell}$,

T. Azemoon ${ }^{p}$, T. Aziz ${ }^{\text {q,r }}$, P.V.K.S. Baba ${ }^{i}$, P. Bagnaia ${ }^{t}$, J.A. Bakken ${ }^{u}$, L. Baksay ${ }^{\mathrm{v}}$, R.C. Ball ${ }^{\mathrm{p}}$,

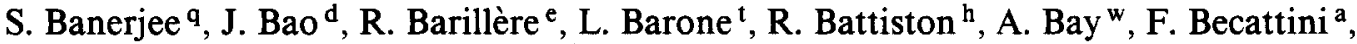
U. Becker ${ }^{\mathrm{k}, \mathrm{j}}$, F. Behner ${ }^{\mathrm{j}}$, J. Behrens ${ }^{\mathrm{j}}, \mathrm{S}$. Beingessner ${ }^{\mathrm{x}}$, Gy.L. Bencze $^{\mathrm{y}}$, J. Berdugo $^{\mathrm{b}}$, P. Berges ${ }^{\mathrm{k}}$, B. Bertucci ${ }^{\text {h }}$, B.L. Betev ${ }^{\mathrm{m}} \mathrm{j}$, M. Biasini ${ }^{\text {h }}$, A. Biland ${ }^{\mathrm{j}}$, G.M. Bilei ${ }^{\mathrm{h}}$, R. Bizzarri ${ }^{\mathrm{t}}$, J.J. Blaising ${ }^{\mathrm{x}}$, B. Blumenfeld ${ }^{d}$, G.J. Bobbink ${ }^{e, z}$, M. Bocciolini ${ }^{a}$, R. Bock ${ }^{r}$, A. Böhm ${ }^{r}$, B. Borgia ${ }^{t}$, D. Bourilkov $^{\text {aa }}$, M. Bourquin ${ }^{w}$, D. Boutigny ${ }^{x}$, B. Bouwens ${ }^{z}$, E. Brambilla ${ }^{f}$, J.G. Branson $^{\text {ab }}$, I.C. Brock ${ }^{\text {ac }}$, M. Brooks ${ }^{\text {ad }}$, C. Buisson ${ }^{\text {ae }}$, A. Bujak ${ }^{\text {af }}$, J.D. Burger ${ }^{\text {, }}$, W.J. Burger ${ }^{\text {w }}$, J.P. Burq ${ }^{\text {ae }}$, J. Busenitz ${ }^{\text {}}$, X.D. Cai ${ }^{\mathrm{i}}$, M. Capell ${ }^{\mathrm{ag}}$, M. Caria ${ }^{\mathrm{h}}$, G. Carlino ${ }^{\mathrm{f}}$, F. Carminati ${ }^{\text {a }}$, A.M. Cartacci ${ }^{\mathrm{a}}$, M. Cerrada ${ }^{\text {b }}$, F. Cesaroni ${ }^{t}$, Y.H. Chang ${ }^{k}$, U.K. Chaturvedi ${ }^{i}$, M. Chemarin ${ }^{\text {ae }}$, A. Chen ${ }^{\text {ah }}$, C. Chen ${ }^{\text {ai }}$, G.M. Chen ${ }^{\text {ai }}$, H.F. Chen ${ }^{\text {aj }}$, H.S. Chen ${ }^{\text {ai }}$, J. Chen ${ }^{k}$, M. Chen ${ }^{k}$, M.L. Chen ${ }^{p}$, W.Y. Chen ${ }^{i}$, G. Chiefari ${ }^{f}$, C.Y. Chien ${ }^{d}$, M. Chmeissani ${ }^{p}$, S. Chung ${ }^{k}$, C. Civinini ${ }^{a}$, I. Clare $^{k}$, R. Clare ${ }^{k}$, T.E. Coan ${ }^{\text {ad }}$, H.O. Cohn ${ }^{\text {ak }}$, G. Coignet ${ }^{x}$, N. Colino ${ }^{\mathrm{e}}$, A. Contin ${ }^{n}$, F. Crijns ${ }^{\text {aa }}$, X.T. Cui ${ }^{i}$, X.Y. Cui ${ }^{i}$, T.S. Dai ${ }^{k}$, R. D'Alessandro ${ }^{a}$, R. de Asmundis ${ }^{f}$, A. Degré ${ }^{x}$, K. Deiters ${ }^{k}$, E. Dénes ${ }^{y}$, P. Denes ${ }^{u}$, F. DeNotaristefani ${ }^{t}$, M. Dhina ${ }^{j}$, D. DiBitonto ${ }^{v}$, M. Diemoz ${ }^{t}$, H.R. Dimitrov ${ }^{m}$, C. Dionisi ${ }^{\text {t,e }}$, M.T. Dova ${ }^{i}$, E. Drago $^{f}$, T. Driever ${ }^{\text {aa }}$, D. Duchesneau ${ }^{\text {w, }}$ P. Duinker ${ }^{z}$, I. Duran ${ }^{a \ell}$, H. El Mamouni ae, A. Engler ${ }^{\text {ac }}$, F.J. Eppling ${ }^{\text {k }}$, F.C. Erné ${ }^{z}$, P. Extermann ${ }^{\text {w }}$, R. Fabbretti ${ }^{\text {am }}$, M. Fabre ${ }^{\text {am }}$, S. Falciano ${ }^{t}$, S.J. Fan ${ }^{\text {an }}$, O. Fackler ${ }^{\text {ag }}$, J. Fay ${ }^{\text {ae }}$, M. Felcini ${ }^{\mathrm{e}}$, T. Ferguson ${ }^{\text {ac }}$, D. Fernandez ${ }^{\mathrm{b}}$, G. Fernandez ${ }^{\mathrm{b}}$, F. Ferroni $^{\mathrm{t}}$, H. Fesefeldt $^{\mathrm{r}}$, E. Fiandrini ${ }^{\text {h }}$, J. Field ${ }^{\text {w }}$, F. Filthaut ${ }^{\text {aa }}$, G. Finocchiaro ${ }^{t}$, P.H. Fisher ${ }^{\text {d }}$, G. Forconi ${ }^{\text {, }}$, T. Foreman ${ }^{z}$, K. Freudenreich ${ }^{j}$, W. Friebel ${ }^{\text {ao }}$, M. Fukushima ${ }^{k}$, M. Gailloud ${ }^{\text {ap }}$, Yu. Galaktionov ${ }^{\text {o,k }}$, E. Gallo ${ }^{a}$, S.N. Ganguli ${ }^{q}$, P. Garcia-Abia ${ }^{b}$, S.S. Gau ${ }^{\text {ah }}$, D. Gele ${ }^{\text {ae }}$, S. Gentile ${ }^{\mathrm{t}, \mathrm{e}}, \mathrm{S}$. Giagu ${ }^{\mathrm{t}}$, S. Goldfarb ${ }^{\mathrm{g}}$, Z.F. Gong ${ }^{\text {aj }}$, E. Gonzalez ${ }^{\mathrm{b}}$, P. Göttlicher ${ }^{\mathrm{r}}$, A. Gougas ${ }^{\mathrm{d}}$, D. Goujon ${ }^{w}$, G. Gratta ${ }^{\text {aq }}$, C. Grinnell ${ }^{\mathrm{k}}$, M. Gruenewald ${ }^{\mathrm{aq}}$, C. Gu ${ }^{\mathrm{i}}$, M. Guanziroli ${ }^{\mathrm{i}}$, J.K. Guo an , V.K. Gupta ${ }^{\text {u }}$, A. Gurtu ${ }^{\text {e,q }}$, H.R. Gustafson ${ }^{p}$, L.J. Gutay ${ }^{\text {af }}$, K. Hangarter ${ }^{r}$, A. Hasan ${ }^{i}$, D. Hauschildt ${ }^{\mathrm{z}}$, C.F. He ${ }^{\text {an }}$, T. Hebbeker ${ }^{\mathrm{r}}$, M. Hebert ${ }^{\text {ab }}$, G. Herten ${ }^{\mathrm{k}}$, U. Herten ${ }^{\mathrm{r}}$,

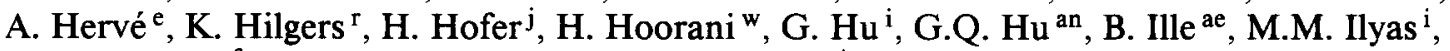
V. Innocente ${ }^{e, f}$, H. Janssen ${ }^{e}$, S. Jezequel ${ }^{x}$, B.N. Jin ${ }^{\text {ai }}$, L.W. Jones ${ }^{p}$, A. Kasser ${ }^{\text {ap }}$, R.A. Khan ${ }^{i}$, Yu. Kamyshkov ${ }^{\text {ak }}$, P. Kapinos ${ }^{\ell, a o}$, J.S. Kapustinsky ad, Y. Karyotakis ${ }^{\text {a, }}$, M. Kaur ${ }^{i}$, S. Khokhar ${ }^{i}$, M.N. Kienzle-Focacci ${ }^{\text {w }}$,W.W. Kinnison ${ }^{\text {ad }}$, D. Kirkby ${ }^{\text {aq }}$, S. Kirsch ${ }^{\text {ao }}$, W. Kittel ${ }^{\text {aa }}$, A. Klimentov ${ }^{\text {k,o }}$, A.C. König aa ${ }^{\text {aa }}$ E. Koffeman ${ }^{z}$, O. Kornadt ${ }^{r}$, V. Koutsenko ${ }^{\text {k,o }}$, A. Koulbardis ${ }^{\ell}$, R.W. Kraemer ${ }^{\text {ac }}$, T. Kramer ${ }^{\text {k }}$, V.R. Krastev ${ }^{\text {m,h }}$, W. Krenz ${ }^{\text {r }}$, A. Krivshich ${ }^{\ell}$, H. Kuijten ${ }^{\text {aa }}$, K.S. Kumar ${ }^{\text {ar }}$, A. Kunin ${ }^{\text {ar,o }}$, G. Landi ${ }^{a}$, D. Lanske ${ }^{r}$, S. Lanzano ${ }^{f}$, P. Lebrun ${ }^{\text {ae }}$, P. Lecomte ${ }^{j}$, P. Lecoq ${ }^{\text {e }}$, P. Le Coultre ${ }^{\text {j }}$, D.M. Lee ${ }^{\text {ad }}$, I. Leedom ${ }^{\text {g }}$, J.M. Le Goff ${ }^{\text {, }}$, R. Leiste ${ }^{\text {ao }}$, M. Lenti ${ }^{\text {a }}$, E. Leonardi ${ }^{t}$, J. Lettry ${ }^{\mathrm{j}}$, X. Leytens ${ }^{\mathrm{z}}$, C. Li ${ }^{\text {aj,i }}$, H.T. Li ${ }^{\text {ai }}$, P.J. Li ${ }^{\text {an }}$, X.G. Li ${ }^{\text {ai }}$, J.Y. Liao $^{\text {an }}$, W.T. Lin ${ }^{\text {ah }}$, Z.Y. Lin aj, F.L. Linde ${ }^{e, z}$, B. Lindemann ${ }^{r}$, D. Linnhofer ${ }^{j}$, L. Lista $^{f}$, Y. Liu ${ }^{i}$, W. Lohmann ${ }^{\text {ao,e }}$, E. Longo ${ }^{t}$, Y.S. Lu ${ }^{\text {ai }}$, J.M. Lubbers ${ }^{\mathrm{e}}$, K. Lübelsmeyer ${ }^{\mathrm{r}}$, C. Luci ${ }^{\mathrm{t}}$, D. Luckey ${ }^{\mathrm{n}, \mathrm{k}}$, 
L. Ludovici $^{\mathrm{t}}$, L. Luminari ${ }^{\mathrm{t}}$, W. Lustermann ${ }^{\text {ao }}$, J.M. Ma ${ }^{\text {ai }}$, W.G. Ma ${ }^{\text {aj }}$, M. MacDermott ${ }^{\mathrm{j}}$,

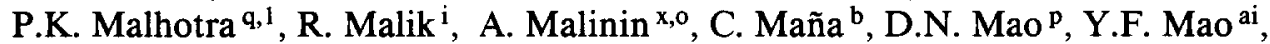

M. Maolinbay ${ }^{j}$, P. Marchesini ${ }^{j}$, F. Marion ${ }^{\text {x }}$, A. Marin ${ }^{\text {c }}$, J.P. Martin ${ }^{\text {ae }}$, L. Martinez-Laso ${ }^{b}$,

F. Marzano ${ }^{\mathfrak{t}}$, G.G.G. Massaro ${ }^{z}$, T. Matsuda ${ }^{\text {k }}$, K. Mazumdar ${ }^{\text {q }}$, P. McBride ${ }^{\text {ar }}$, T. McMahon ${ }^{\text {af }}$,

D. McNally ${ }^{j}$, Th. Meinholz ${ }^{\mathrm{r}}$, M. Merk ${ }^{\text {aa }}$, L. Merola ${ }^{\mathrm{f}}$, M. Meschini ${ }^{\mathrm{a}}$, W.J. Metzger ${ }^{\text {aa }}$, Y. Mi $^{\mathrm{i}}$, G.B. Mills ${ }^{\text {ad }}$, Y. Mir ${ }^{\mathrm{i}}$, G. Mirabelli ${ }^{\mathrm{t}}$, J. Mnich ${ }^{\mathrm{r}}$, M. Möller ${ }^{\mathrm{r}}$, B. Monteleoni ${ }^{\mathrm{a}}, \mathrm{R}$. Morand ${ }^{\mathrm{x}}$, S. Morganti ${ }^{t}$, N.E. Moulai ${ }^{i}$, R. Mount ${ }^{\text {aq }}$, S. Müller ${ }^{r}$, A. Nadtochy ${ }^{\ell}$, E. Nagy ${ }^{y}$, M. Napolitano ${ }^{f}$, H. Newman ${ }^{\text {aq }}$, C. Neyer ${ }^{j}$, M.A. Niaz ${ }^{i}$, A. Nippe ${ }^{r}$, H. Nowak ${ }^{\text {ao }}$, G. Organtini ${ }^{t}$, D. Pandoulas ${ }^{r}$,

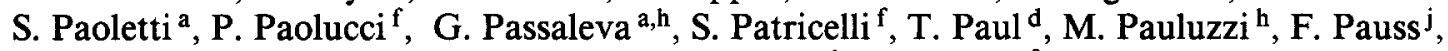
Y.J. Pei ${ }^{r}$, D. Perret-Gallix ${ }^{x}$, J. Perrier ${ }^{\text {w }}$, A. Pevsner ${ }^{\text {d }}$, D. Piccolo ${ }^{\text {f }}$, M. Pieri e,a, P.A. Piroué ${ }^{u}$, F. Plasil ${ }^{\text {ak }}$, V. Plyaskin ${ }^{\circ}$, M. Pohl ${ }^{j}$, V. Pojidaev ${ }^{\text {o,a }}$, N. Produit ${ }^{w}$, J.M. Qian $^{p}$, K.N. Qureshi ${ }^{i}$, R. Raghavan ${ }^{\mathrm{q}}$, G. Rahal-Callot ${ }^{\mathrm{j}}$, G. Raven ${ }^{\mathrm{z}}$, P. Razis $^{\text {as }}$, K. Read $^{\text {ak }}$, D. Ren $^{\mathrm{j}}, \mathrm{Z}$ Ren $^{\mathrm{i}}$, M. Rescigno ${ }^{t}$, S. Reucroft ${ }^{\mathrm{g}}$, A. Ricker $^{\mathrm{r}}$, S. Riemann ${ }^{\text {ao }}$, O. Rind ${ }^{\text {p }}$, H.A. Rizvi $^{\mathrm{i}}$, F.J. Rodriguez ${ }^{\mathrm{b}}$, B.P. Roe ${ }^{\mathrm{p}}$, M. Röhner ${ }^{\mathrm{r}}$, S. Röhner ${ }^{\mathrm{r}}$, L. Romero ${ }^{\mathrm{b}}$, J. Rose ${ }^{\mathrm{r}}$, S. Rosier-Lees ${ }^{\mathrm{x}}$, R. Rosmalen ${ }^{\text {aa }}$, Ph. Rosselet ${ }^{\text {ap }}$, A. Rubbia ${ }^{k}$, J.A. Rubio ${ }^{\mathrm{e}}$, H. Rykaczewski ${ }^{\mathrm{j}}$, M. Sachwitz $^{\text {ao }}$, E. Sajan $^{\mathrm{h}}$, J. Salicio $^{\text {e }}$, J.M. Salicio ${ }^{\text {, }}$, G.S. Sanders ${ }^{\text {ad }}$, A. Santocchia ${ }^{\text {h }}$, M.S. Sarakinos ${ }^{\text {k }}$, G. Sartorelli ${ }^{\text {n,i }}$, M. Sassowsky ${ }^{r}$, G. Sauvage ${ }^{x}$, V. Schegelsky ${ }^{\ell}$, K. Schmiemann ${ }^{r}$, D. Schmitz ${ }^{r}$, P. Schmitz ${ }^{r}$, M. Schneegans ${ }^{x}$, H. Schopper ${ }^{\text {at }}$, D.J. Schotanus ${ }^{\text {aa }}$, S. Shotkin ${ }^{k}$, H.J. Schreiber ${ }^{\text {ao }}$, J. Shukla ${ }^{\text {ac }}$, R. Schulte ${ }^{\mathrm{r}}$, S. Schulte ${ }^{\mathrm{r}}$, K. Schultze ${ }^{\mathrm{r}}$, J. Schütte ${ }^{\text {ar }}$, J. Schwenke ${ }^{r}$, G. Schwering ${ }^{r}$, C. Sciacca ${ }^{\mathrm{f}}$, I. Scott $^{\text {ar }}$, R. Sehgal ${ }^{\text {i }}$, P.G. Seiler ${ }^{\text {am }}$, J.C. Sens ${ }^{\text {e,z }}$, L. Servoli ${ }^{\text {h }}$, I. Sheer $^{\text {ab }}$, D.Z. Shen ${ }^{\text {an }}$, S. Shevchenko aq, X.R. Shi ${ }^{\text {aq }}$, E. Shumilov ${ }^{\circ}$, V. Shoutko ${ }^{\circ}$, E. Soderstrom ${ }^{\text {u }}$, A. Sopczak ${ }^{\text {ab }}$, C. Spartiotis ${ }^{d}$, T. Spickermann ${ }^{r}$, P. Spillantini ${ }^{a}$, R. Starosta ${ }^{r}$, M. Steuer ${ }^{\text {n,k }}$, D.P. Stickland ${ }^{u}$, F. Sticozzi ${ }^{k}$, H. Stone ${ }^{\text {w }}$, K. Strauch ${ }^{\text {ar }}$, B.C. Stringfellow ${ }^{\text {af }}$, K. Sudhakar ${ }^{\text {q,r }}$, G. Sultanov ${ }^{i}$, R.L. Sumner ${ }^{u}$, L.Z. Sun ${ }^{\text {aj,i }}$, H. Suter ${ }^{j}$, R.B. Sutton ${ }^{a c}$, J.D. Swain ${ }^{i}$, A.A. Syed ${ }^{i}$, X.W. Tang ${ }^{a i}$, L. Taylor $^{\mathrm{g}}$, C. Timmermans ${ }^{\mathrm{aa}}$, Samuel C.C. Ting ${ }^{\mathrm{k}}$, S.M. Ting ${ }^{\mathrm{k}}$, M. Tonutti ${ }^{\mathrm{r}}$, S.C. Tonwar $^{\mathrm{q}}$, J. Tóth ${ }^{\mathrm{y}}$, A. Tsaregorodtsev ${ }^{\ell}$, G. Tsipolitis ${ }^{\mathrm{ac}}$, C. Tully ${ }^{\mathrm{aq}}$, K.L. Tung ${ }^{\text {ai }}$, J. Ulbricht ${ }^{\mathrm{j}}$, L. Urbán ${ }^{\mathrm{y}}$,

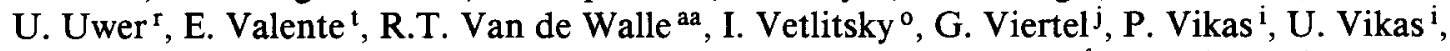
M. Vivargent ${ }^{x}$, H. Vogel ${ }^{\text {ac }}$, H. Vogt ${ }^{\text {ao }}$, I. Vorobiev ${ }^{\circ}$, A.A. Vorobyov ${ }^{\ell}$, L. Vuilleumier $^{\text {ap }}$, M. Wadhwa ${ }^{\text {i }}$, W. Wallraffr ${ }^{r}$, C.R. Wang ${ }^{\text {aj }}$, G.H. Wang ${ }^{\text {ac }}$, J.H. Wang ${ }^{\text {ai }}$, Q.F. Wang ${ }^{\text {ar }}$, X.L. Wang aj, Y.F. Wang ${ }^{a}$, Z.M. Wang ${ }^{i, a j}$, A. Weber ${ }^{r}$, J. Weber ${ }^{j}$, R. Weill ${ }^{\text {ap }}$, T.J. Wenaus ${ }^{\text {ag }}$, J. Wenninger $^{\text {w }}$, M. White ${ }^{k}$, C. Willmott ${ }^{b}$, F. Wittgenstein ${ }^{e}$, D. Wright ${ }^{\text {u }}$, R.J. Wu ${ }^{\text {ai }}$, S.X. Wu ${ }^{i}$, Y.G. Wu ${ }^{\text {ai }}$, B. Wysłouch ${ }^{k}$, Y.Y. Xie an, Y.D. Xu ${ }^{\text {ai }}$, Z.Z. Xu ${ }^{\text {aj }}$, Z.L. Xue ${ }^{\text {an }}$, D.S. Yan ${ }^{\text {an }}$, X.J. Yank ${ }^{k}$, B.Z. Yang ${ }^{\text {aj }}$, C.G. Yang ${ }^{\text {ai }}$, G. Yang ${ }^{\mathrm{i}}$, K.S. Yang ${ }^{\text {ai }}$, Q.Y. Yang ${ }^{\text {ai }}$, Z.Q. Yang ${ }^{\text {an }}$, C.H. Ye ${ }^{i}$, J.B. Ye ${ }^{\text {aj }}$, Q. Ye ${ }^{i}$, S.C. Yeh ${ }^{\text {ah }}$, Z.W. Yin ${ }^{\text {an }}$, J.M. You ${ }^{i}$, N. Yunus ${ }^{i}$, M. Yzerman ${ }^{z}$, C. Zaccardelli ${ }^{\text {aq }}$, P. Zemp ${ }^{\text {j, M. }}$ Zeng ${ }^{\mathrm{i}}$, Y. Zeng ${ }^{\mathrm{r}}$, D.H. Zhang ${ }^{\mathrm{z}}$, Z.P. Zhang aj,i, B. Zhou ${ }^{\mathrm{c}}$, J.F. Zhou ${ }^{\mathrm{r}}$, R.Y. Zhu ${ }^{\text {aq }}$, H.L. Zhuang ${ }^{\text {ai }}$, A. Zichichi ${ }^{\mathrm{n}, \mathrm{e}, \mathrm{i}}$ and B.C.C. van der Zwaan ${ }^{\mathrm{z}}$

a INFN - Sezione di Firenze and Università di Firenze, I-50125 Florence, Italy

b Centro de Investigaciones Energeticas, Medioambientales y Tecnologicas, CIEMAT, E-28040 Madrid, Spain

c Boston University, Boston, MA 02215, USA

d Johns Hopkins University, Baltimore, MD 21218, USA

e European Laboratory for Particle Physics, CERN, CH-1211 Geneva 23, Switzerland

f INFN - Sezione di Napoli and Università di Napoli, I-80125 Naples, Italy

g Northeastern University, Boston, MA 02115, USA

h INFN - Sezione di Perugia and Università Degli Studi di Perugia, I-06100 Perugia, Italy

i World Laboratory, FBLJA Project, CH-1211 Geneva 23, Switzerland

j Eidgenössische Technische Hochschule, ETH Zürich, CH-8093 Zurich, Switzerland

k Massachusetts Institute of Technology, Cambridge, MA 02139, USA

${ }^{\ell}$ Nuclear Physics Institute, St. Petersburg, Russian Federation 
m Bulgarian Academy of Sciences, Institute of Mechatronics, BU-1113 Sofia, Bulgaria

n INFN - Sezione di Bologna, 1-40126 Bologna, Italy

- Institute of Theoretical and Experimental Physics, ITEP, 117259 Moscow, Russian Federation

p University of Michigan, Ann Arbor, MI 48109, USA

q Tata Institute of Fundamental Research, Bombay 400 005, India

r I. Physikalisches Institut, RWTH, W-5100 Aachen, FRG ${ }^{2}$ and III. Physikalisches Institut, RWTH, W-5100 Aachen, FRG ${ }^{2}$

'INFN - Sezione di Roma and Università di Roma "La Sapienza", I-00185 Rome, Italy

"Princeton University, Princeton, NJ 08544, USA

$\checkmark$ University of Alabama, Tuscaloosa, AL 35486, USA

w University of Geneva, $\mathrm{CH}-1211$ Geneva 4, Switzerland

${ }^{x}$ Laboratoire de Physique des Particules, LAPP, F-74941 Annecy-le-Vieux, France

y Central Research Institute for Physics of the Hungarian Academy of Sciences, H-1525 Budapest 114, Hungary

z National Institute for High Energy Physics, NIKHEF, NL-1009 DB Amsterdam, The Netherlands

aa University of Nijmegen and NIKHEF, NL-6525 ED Nijmegen, The Netherlands

ab University of California, San Diego, CA 92182, USA

ac Carnegie Mellon University, Pittsburgh, PA 15213, USA

ad Los Alamos National Laboratory, Los Alamos, NM 87544, USA

ae Institut de Physique Nucléaire de Lyon, IN2P3-CNRS/Université Claude Bernard, F-69622 Villeurbanne Cedex, France

af Purdue University, West Lafayette, IN 47907, USA

ag Lawrence Livermore National Laboratory, Livermore, CA 94550, USA

ah High Energy Physics Group, Taiwan, ROC

ai Institute of High Energy Physics, IHEP, Beijing, China

aj Chinese University of Science and Technology, USTC, Hefei, Anhui 230 029, China

ak Oak Ridge National Laboratory, Oak Ridge, TN 37830, USA

a Departamento de Fisica de Particulas Elementales, Universidad de Santiago, E-15706 Santiago de Compostela, Spain

am Paul Scherrer Institut, PSI, CH-5232 Villigen, Switzerland

an Shanghai Institute of Ceramics, SIC, Shanghai, China

ao DESY-Institut für Hochenergiephysik, O-1615 Zeuthen, FRG

ap University of Lausanne, CH-1015 Lausanne, Switzerland

aq California Institute of Technology, Pasadena, CA 91125, USA

ar Harvard University, Cambridge, MA 02139, USA

as Department of Natural Sciences, University of Cyprus, Nicosia, Cyprus

at University of Hamburg, W-2000 Hamburg, FRG

Received 27 July 1992

We have measured the cross-section of the production of single photon events in $\mathrm{e}^{+} \mathrm{e}^{-}$collisions near the $\mathrm{Z}^{0}$ resonance. For an integrated luminosity of $9.6 \mathrm{pb}^{-1}$, we have observed 202 single photon candidates with energy between 0.9 and $3.5 \mathrm{GeV}$ in the polar angular region between $45^{\circ}$ and $135^{\circ}$. Assuming that the only stable weakly interacting particles are light neutrinos with standard model couplings, we determine the number of light neutrino species to be $N_{\nu}=3.14 \pm 0.24$ (stat.) \pm 0.12 (syst.). This corresponds to an invisible $\mathrm{Z}^{0}$ width of $\Gamma_{\mathrm{inv}}=524 \pm 40 \pm 20 \mathrm{MeV}$.

\section{Introduction}

The number of fermion generations, which can be determined from the number of light neutrinos, is among the important questions in the standard model.

1 Deceased.

2 Supported by the German Bundesministerium für Forschung und Technologie.
In the standard model the decay width of the $\mathrm{Z}^{0}$ into each neutrino family is calculated to be $\Gamma_{\nu \bar{\nu}}=166.8 \pm$ $1.5 \mathrm{MeV}$ where the uncertainty corresponds to a variation of the top mass between 90 and $250 \mathrm{GeV}[1,2]$. Additional generations or other new weakly interacting particles with masses below $\frac{1}{2} M_{Z}$, would lead to a decay width of the $Z^{0}$ into invisible channels larger than the standard model prediction for three families while a smaller value could be produced, for exam- 


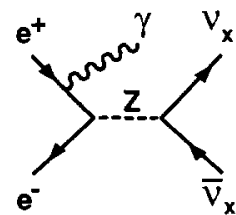

a) b)

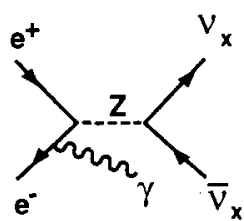

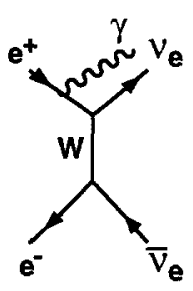

c)

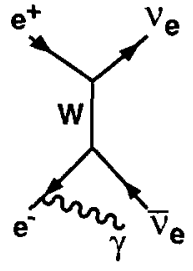

d)

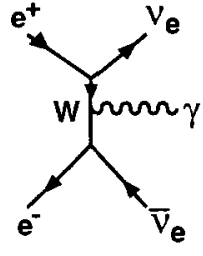

e)
Fig. 1. Lowest order Feynman diagrams contributing to $\mathrm{e}^{+} \mathrm{e}^{-} \rightarrow \nu \bar{\nu} \gamma$.

ple, by the presence of one or more right-handed neutrinos mixed with the left-handed ones [3]. Thus the number of light neutrino generations $N_{\nu}$, defined as the ratio between the measured invisible decay width of the $Z^{0}, \Gamma_{\mathrm{inv}}$, and the standard model expectation $\Gamma_{\nu \bar{\nu}}$ for each neutrino family, need not to be an integer number and has to be measured with the highest possible accuracy.

An indirect determination of $N_{\nu}$ has been made at LEP through the analysis of the $Z^{0}$ lineshape, subtracting from the total width the visible partial ones. The latest result obtained by $\mathrm{L} 3$ in this approach is $N_{\nu}=3.05 \pm 0.10$ [4] where the errors come mainly from the uncertainty in the luminosity measurement and the selection of the charged leptonic and hadronic final states.

A direct method for measuring the $Z^{0}$ width into neutrinos, and thus for counting the number of light neutrino types, is based on the measurement of the cross-section for the radiative process $\mathrm{e}^{+} \mathrm{e}^{-} \rightarrow \nu \bar{\nu} \gamma$ [5]. The signature of such events is a single photon arising from initial state radiation. The diagrams which contribute to this process at tree level are shown in fig. 1 .

Near the $Z^{0}$ resonance, the square of the $s$-channel
$\mathrm{Z}^{0}$ exchange terms (figs. 1a, 1b), summed over all the neutrino types, gives the main contribution to the cross-section and is proportional to $N_{\nu}$. In the energy range scanned by LEP around the $Z^{0}$ peak, the square of the $t$-channel W exchange terms (figs. $1 \mathrm{c}-1 \mathrm{e}$ ) and their interference with the $\mathrm{Z}^{0}$ terms contributes less than $3 \%$ to the total cross-section for three neutrino species. The contribution of these terms is independent of $N_{\nu}$ because they give rise only to $\nu_{\mathrm{e}} \bar{\nu}_{\mathrm{e}}$ pairs.

The differential cross-section can be written as

$\frac{\mathrm{d}^{2} \sigma}{\mathrm{d} E_{\gamma} \mathrm{d} \cos \theta_{\gamma}}=H\left(E_{\gamma}, \cos \theta_{\gamma}, s\right) \sigma_{0}\left(s^{\prime}\right)$,

where $H$ is a radiator function describing the initial state radiation of a photon of energy $E_{\gamma}$ at an angle $\theta_{\gamma}$ with respect to the beam axis, $s$ is the square of the center of mass energy, and $\sigma_{0}\left(s^{\prime}\right)$ is the cross-section for the process $\mathrm{e}^{+} \mathrm{e}^{-} \rightarrow \nu \bar{\nu}$, at the "reduced" center of mass energy given by $s^{\prime}=s\left(1-2 E_{\gamma} / \sqrt{s}\right)$.

The measurement is optimally carried out at energies at least $3 \mathrm{GeV}$ above the $Z^{0}$ mass where the ratio between the signal and QED background processes is maximal and the full width of the $\mathrm{Z}^{0}$ resonance is exploited $[1,6]$. However, the LEP line-shape scanning at the $Z^{0}$ resonance [7] has resulted in less favourable conditions for our measurements, requiring a trigger efficient for low energy photons $\left(E_{\gamma}>1 \mathrm{GeV}\right)$. Around the $\mathrm{Z}^{0}$ pole, photons from $\mathrm{e}^{+} \mathrm{e}^{-} \rightarrow \nu \bar{\nu} \gamma$ have low energies with a rapidly falling spectrum. The L3 electromagnetic calorimeter is well suited for the detection of such photons, providing an energy resolution better than $1.5 \%$ for $E_{\gamma}>2 \mathrm{GeV}$ in combination with an uncertainty on the absolute energy scale smaller than $4 \mathrm{MeV}$.

A first measurement of the $\nu \bar{\nu} \gamma$ cross-section based on data taken at LEP in 1990 has been already published by the OPAL [8] and L3 [9] Collaboration. In this paper, we present results from data taken in 1991 at center of mass energies between 88.6 and $93.8 \mathrm{GeV}$ with a corresponding integrated luminosity of $9.6 \mathrm{pb}^{-1}$. With respect to the analysis of the 1990 data, we have the benefit of three major improvements:

(1) an increased integrated luminosity: from 2.9 to $9.6 \mathrm{pb}^{-1}$;

(2) a lower trigger threshold: from 1.5 to $0.9 \mathrm{GeV}$;

(3) a dedicated trigger on isolated electrons from $\mathrm{e}^{+} \mathrm{e}^{-} \rightarrow \mathrm{e}^{+} \mathrm{e}^{-} \gamma$ providing a measurement of the single 
photon trigger efficiency.

\section{The $\mathbf{L 3}$ detector}

The L3 detector at LEP covers $99 \%$ of the full solid angle. It is designed to measure energy and position of leptons and photons with high precision. A detailed description of the detector and its performance can be found elsewhere [10] and here we only outline the features which are relevant to the present analysis.

The detector consists of a time expansion chamber (TEC) for the tracking and vertex reconstruction of charged particles, a high resolution electromagnetic calorimeter made of about 11000 bismuth germanium oxide (BGO) crystals, a hadron calorimeter (HCAL) with uranium absorber and brass proportional wire chambers and a high precision muon spectrometer, consisting of three layers of multi-wire drift chambers, which measures the muon trajectory 56 times in the bending plane (by the P-chambers) and 8 times in the non-bending direction (by the Z-chambers). A cylindrical array of 30 scintillation counters is installed in the barrel region between the BGO and the HCAL. All these detectors are inside a $12 \mathrm{~m}$ inner diameter solenoidal magnet which provides a uniform magnetic field of $0.5 \mathrm{~T}$ along the beam direction.

The polar angle acceptance of the BGO barrel extends from $42.3^{\circ}$ to $137.7^{\circ}$ and is fully covered by the TEC. The BGO endcaps cover from $11.4^{\circ}$ to $35.2^{\circ}$ and from $144.8^{\circ}$ to $168.6^{\circ}$, the HCAL from $6^{\circ}$ to $174^{\circ}$ and the muon spectrometer from $36^{\circ}$ to $144^{\circ}$.

The minimum angle at which particles can be detected, critical to the suppression of QED background, is defined by the luminosity monitors. They consist of two electromagnetic calorimeters and two sets of proportional wire chambers, situated symmetrically on either side of the interaction point. Each calorimeter is a finely segmented and azimuthally symmetric array of 304 BGO crystals covering the polar angular range $24.93<\theta<69.94 \mathrm{mrad}$. The energy resolution of the calorimeters is about $2 \%$ at $45 \mathrm{GeV}$, and the position resolution is $0.4 \mathrm{mrad}$ in $\theta$ and $8.7 \mathrm{mrad}$ in $\phi$.

Apart from the region below the minimum detection angle, there is a region (hole) about $2^{\circ}$ wide in $\theta$ between the luminosity monitors and the hadron calorimeter endcaps which is not covered by any de- tector. There is thus additional background from radiative Bhabha events when one of the particles escapes undetected through this hole. Moreover up to about $9^{\circ}$ the efficiency of the hadron calorimeter, for electrons and photons, is very poor because of two small lead rings installed in front of it in July 1991 to shield our tracking chamber from the beam halo.

The response of the L3 detector is modelled using the GEANT3 [11] detector simulation program which includes the effects of energy loss, multiple scattering and showering in the detector materials and in the beam pipe. Hadronic showers in the calorimeters are simulated with the GHEISHA [12] program. For each of the physical processes studied below, Monte Carlo events are passed through the detector simulation program and are reconstructed by the same program used to reconstruct the real ones.

\section{Single photon trigger}

The single photon trigger is entirely based on the BGO barrel. Each crystal has a separate output used for first level trigger purposes while the full digitized event is available to the third level trigger.

These outputs are summed in trigger segments of 30 crystals each, with a granularity of 32 segments in $\phi$ and 8 in $\theta$. These 256 sums are digitised by the trigger FERA (Fast Encoding Readout ADC) and sent to the first level energy trigger processors which add the trigger signals at constant $\theta$ and at constant $\phi$ to obtain 32 sums $\Sigma_{\theta}$ and 8 sums $\Sigma_{\phi}$ respectively [13]. In order to suppress the contribution from coherent noise, the calculation includes only trigger segments above a $900 \mathrm{MeV}$ threshold (called bias in the following).

The first level single photon trigger requires the following conditions:

$\Sigma_{\theta}^{\max }$ and $\sum_{\phi}^{\max }>900 \mathrm{MeV}$,

$\Sigma_{\theta}^{\max }$ and $\Sigma_{\phi}^{\max }>0.8 \Sigma_{\text {tot }}$,

where $\Sigma_{\theta}^{\max }$ and $\Sigma_{\phi}^{\max }$ are respectively the largest $\theta$ and $\phi$ energy sum and $\Sigma_{\text {tot }}$ is the total energy seen by the trigger in the BGO barrel. In 1991, the first level single photon trigger rate was about $1 \mathrm{~Hz}$, mainly due to electronic noise.

The second level trigger does not apply any rejection for this channel, while the third level trigger requires 


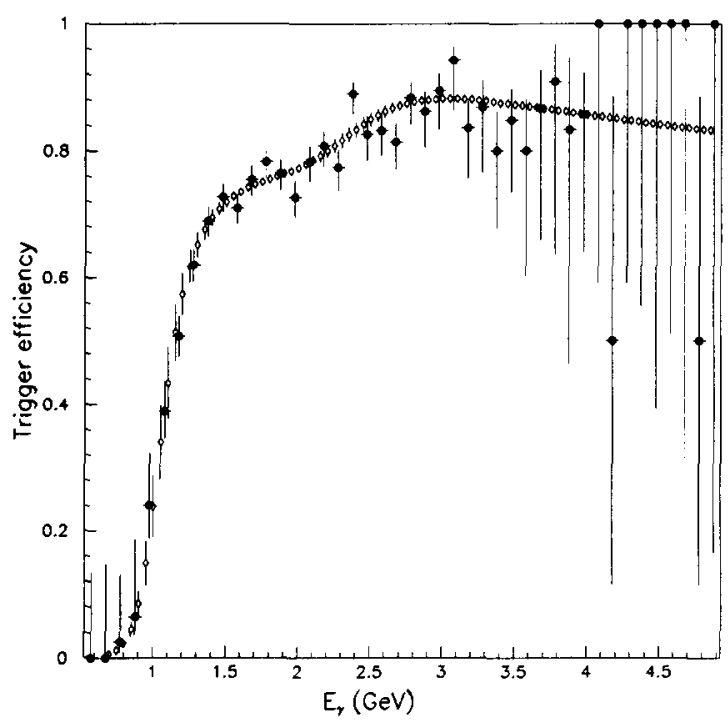

Fig. 2. Trigger efficiency curve as a function of the photon energy.

a cluster in the BGO barrel with energy greater than $500 \mathrm{MeV}$ shared by more than 2 and less than 80 crystals. The typical output rate on tape under these trigger conditions was about $0.01 \mathrm{~Hz}$. The third level trigger efficiency in the acceptance region exceeds $99.9 \%$ for photons above $900 \mathrm{MeV}$ apart from geometrical inefficiency due to dead crystals. This is taken into account in the determination of the event selection efficiency.

We measure the single photon trigger efficiency in two ways.

In the first, we use a sample of radiative Bhabha events with an isolated electron in the BGO barrel. These events are collected under an independent trigger which requires a coincidence between a cluster in one of the luminosity monitors and a large angle track in the vertex chamber. The single photon trigger efficiency is then measured by checking whether or not this trigger is also activated. The dark circles in fig. 2 plot the efficiency found as a function of the photon energy. The slight dip at $2 \mathrm{GeV}$ is due to $15 \%$ of segments which have a $2 \mathrm{GeV}$ bias. The errors represent $68 \% \mathrm{CL}$ intervals using binomial statistics.

The single electron sample is also useful for checking the Monte Carlo program TEEG [14] which we use to estimate the dominant QED background $\mathrm{e}^{+} \mathrm{e}^{-} \gamma$ when only the photon is observed in the detector.

The second way is based on a Monte Carlo simulation of the trigger algorithm obtained by superimposing the generated single photon on randomly triggered beam gate events. Thus the actual electronic noise of the trigger channels as well as the FERA energy resolution and pedestals fluctuations are taken into account.

The trigger efficiency curve from the Monte Carlo simulation is also shown in fig. 2. The errors correspond to the uncertainties in the model used to compute the efficiency. They include the statistical errors on the FERA resolution and the systematic error in the knowledge of the shower development in the BGO. The trigger efficiencies above $900 \mathrm{MeV}$, averaged over the photon spectra at different $\mathrm{CM}$ energies, range from $59 \%$ at the $Z^{0}$ peak to $72 \%$ at $3 \mathrm{GeV}$ above the peak.

In the following analysis we use the efficiency curve computed from the Monte Carlo. We estimate a systematic error of $1.3 \%$ on the determination of the trigger efficiency by varying the simulated curve within the experimental point errors.

\section{Event selection}

The selection of the $\mathrm{Z}^{0} \rightarrow \nu \bar{\nu} \gamma$ candidates aims at identifying events with (a) a neutral electromagnetic energy deposit in the BGO and (b) no other activity in the detector apart from what is consistent with noise.

The main sources of background are single photon events from QED processes in which all other finalstate particles, mainly produced at small polar angles, escape detection. Among these reactions, the dominant ones are radiative Bhabha scattering $\mathrm{e}^{+} \mathrm{e}^{-} \rightarrow$ $\mathrm{e}^{+} \mathrm{e}^{-} \gamma$, two-photon processes $\mathrm{e}^{+} \mathrm{e}^{-} \rightarrow \mathrm{e}^{+} \mathrm{e}^{-} \mathrm{X}$, where $\mathrm{X}$ is a $\pi^{0}, \eta, \eta^{\prime}, \mathrm{a}_{2}$, for $\ell^{+} \ell^{-} \gamma$ and the process $\mathrm{e}^{+} \mathrm{e}^{-} \rightarrow$ $\gamma \gamma \gamma[1,9]$.

We require the following:

(a1) An energy deposit in the BGO greater than 900 $\mathrm{MeV}$, at a polar angle between $45^{\circ}$ and $135^{\circ}$, shared between three or more crystals.

(a2) The lateral shape of the energy deposit to be consistent with the one expected from a single electromagnetic particle coming from the interaction point.

(b1) No other deposited energy greater than 100 $\mathrm{MeV}$ with three or more crystals in the BGO barrel 
or endcaps.

(b2) No charged tracks found in the TEC.

(b3) Less than $1 \mathrm{GeV}$ deposited in either luminosity monitor.

(b4) Less than $3 \mathrm{GeV}$ deposited in the HCAL.

(b5) No tracks or segments of track seen in the muon spectrometer and no match found between the muon Z-chambers hits and the BGO energy deposit.

Cut (a1) defines our acceptance region for the events and cut $(\mathrm{a} 2)$ ensures that the energy deposit is electromagnetic. Cut (b1) reduces the contamination from two-photon production of resonances decaying in two or more photons, cut (b2) removes the single electron contamination and beam-gas or beamwall events, cuts (b3) and (b4) (in the regions not covered by the $\mathrm{BGO}$ ) reduce the $\mathrm{e}^{+} \mathrm{e}^{-} \gamma$ background.

Cuts (a2), (b1), (b2), (b4) and (b5) remove the contamination from the bremsstrahlung of cosmic rays nearly in time with the beam crossing. Due to the long integration time of the BGO ( $8 \mu$ s starting at $2.8 \mu$ s before the beam crossing), an out of time cosmic emitting a hard photon bremsstrahlung, when only the BGO is active, can simulate a single photon event. To evaluate a possible cosmic contamination from this source, we carried out an independent selection of cosmic ray events and then measured the effect of veto cuts (a2) and (b1) on this sample. The independent selection is based on the BGO segment signals which are used for level 1 triggering. Here we take advantage of the fact that the segment signal is sampled in a gate of $2 \mu$ s chosen so that if the event is in time the trigger will measure the same energy as the digital readout which is reconstructed offline. Thus the ratio between these two values gives an indication of how much out of time the event was. No events on this sample survive all the selection cuts and we estimate the contamination from out of time cosmic rays to be less than 3 events at $95 \% \mathrm{CL}$.

After applying all our selection cuts we obtain a sample of 291 single photon candidates.

The efficiency of the selection cuts (a1) and (a2) computed from Monte Carlo and checked with the single electron sample is 0.92 while the efficiency of the veto cuts (b1) to (b5) is 0.95 . The latter was measured by using randomly triggered beam gate events to study the effects of detector noise and also Monte Carlo events to estimate photon conversion, double initial state radiation and shower leakage from the

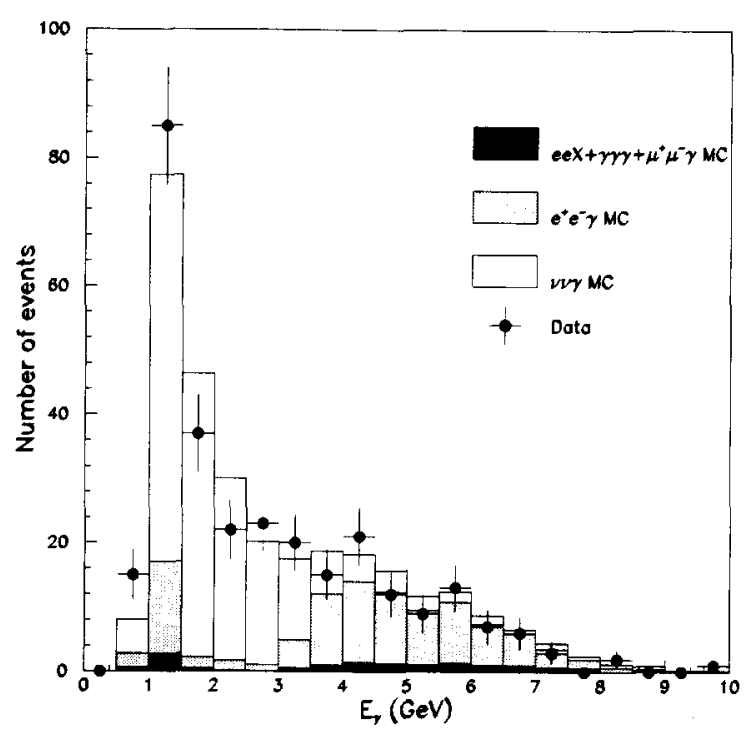

Fig. 3. Energy spectrum of the single photons along with the Monte Carlo predictions (eeX means the background from resonances produced via two-photon processes).

BGO into the HCAL. The total efficiency, which includes the trigger efficiency values given in section 3 , ranges from 0.55 to 0.63 as a function of the center of mass energy.

The energy spectrum of the single photon candidates is shown in fig. 3 together with the Monte Carlo prediction for the signal expected from three light neutrino families and the backgrounds. The main background contribution is due to the $\mathrm{e}^{+} \mathrm{e}^{-} \gamma$ channel, when both electron and positron escape through the beam pipe $\left(E_{\gamma}<1.5 \mathrm{GeV}\right)$ or one of the two goes undetected inside the hole or lead ring region just outside the luminosity monitors $\left(3.0<E_{\gamma}<7.0 \mathrm{GeV}\right)$ while the other stays in the beam pipe. It accounts for 94 events. As a check of the $\mathrm{e}^{+} \mathrm{e}^{-} \gamma$ contamination, we have also compared the number of single-tag single $\gamma$ events. We found 97 events in the data while the TEEG program predicts 73 events. This difference has been taken into account in our systematical error.

Other sources of smaller backgrounds are twophoton production of resonances, $\gamma \gamma \gamma$ and $\mu \mu \gamma$ final states. We estimate the sum of these backgrounds to be 15 events. Backgrounds from $\mathrm{e}^{+} \mathrm{e}^{-} \rightarrow \mathrm{e}^{+} \mathrm{e}^{-} \mathrm{e}^{+} \mathrm{e}^{-} \gamma$ and $\mathrm{e}^{+} \mathrm{e}^{-} \rightarrow \mathrm{e}^{+} \mathrm{e}^{-} \mu^{+} \mu^{-} \gamma$ are found to be negligible. 
Table 1

Luminosity, observed and expected number of events in the energy range $0.9<E_{\gamma}<3.5 \mathrm{GeV}$ and for a polar angle between $45^{\circ}$ and $135^{\circ}$. The last column shows the corrected cross-section for $\mathrm{e}^{+} \mathrm{e}^{-} \rightarrow \nu \bar{\nu} \gamma$ at each center of mass energy.

\begin{tabular}{lcccccc}
\hline$E_{\mathrm{cm}}(\mathrm{GeV})$ & $\mathcal{L}\left(\mathrm{nb}^{-1}\right)$ & $N_{\text {observed }}$ & $N_{\text {expected }}^{\nu \bar{\nu}}$ & $N_{\text {expected }}^{\mathrm{e}^{+} \mathrm{e}^{-} \gamma}$ & $N_{\text {expected }}^{\text {other back. }}$ & $\sigma(\mathrm{pb})$ \\
\hline 88.56 & 671 & 6 & 2.21 & 1.96 & 0.35 & $12_{-5}^{+12}$ \\
89.55 & 772 & 9 & 4.27 & 2.22 & 0.39 & $18_{-6}^{+11}$ \\
90.25 & 632 & 11 & 5.16 & 1.78 & 0.32 & $31_{-8}^{+15}$ \\
91.25 & 5763 & 116 & 92.41 & 14.42 & 2.96 & $37 \pm 4$ \\
92.04 & 635 & 21 & 17.59 & 1.72 & 0.31 & $64_{-12}^{+19}$ \\
93.05 & 678 & 26 & 29.63 & 1.80 & 0.33 & $66_{-12}^{+17}$ \\
93.75 & 419 & 13 & 18.35 & 1.10 & 0.21 & $52_{-12}^{+21}$ \\
total & 9570 & 202 & 169.6 & 25.0 & 4.9 & \\
\hline
\end{tabular}

\section{Results}

The number of neutrino species is extracted from the number of candidates in the energy range $0.9<$ $E_{\gamma}<3.5 \mathrm{GeV}$ where the signal to background ratio is favourable.

For this energy range, table 1 shows the luminosity, the number of candidates and the $\nu \bar{\nu} \gamma$ expectation for three neutrino families computed with the Monte Carlo program NNGSTR [15]. Also shown are the expected background from radiative Bhabha events and the total expected background from the other reactions (two-photon processes, $\mathrm{e}^{+} \mathrm{e}^{-} \rightarrow \gamma \gamma \gamma$ and $\mathrm{e}^{+} \mathrm{e}^{-} \rightarrow \mu^{+} \mu^{-} \gamma$ ).

The last column of table 1 shows the measured cross-sections corrected for acceptance and detector efficiency for the process $\mathrm{e}^{+} \mathrm{e}^{-} \rightarrow \nu \bar{\nu} \gamma$, when one photon with energy above $0.9 \mathrm{GeV}$ is emitted in the polar angle range between $45^{\circ}$ and $135^{\circ}$ without restrictions on possible emission of additional photons. The errors are only statistical and correspond to $68 \% \mathrm{CL}$.

We extract the number of light neutrino families $N_{\nu}$ by performing a maximum likelihood fit to the number of candidates shown in table 1 . We use Poisson probabilities calculated as a function of the expected number of signal plus background events. We compute for each CM energy the cross-section corresponding to different values of $N_{\nu}$ between 2 and 4 and use a straight line fit to get a parametrisation of the cross-section dependence on $N_{\nu}$. We use an improved Born approximation of the analytical calculation of ref. [16], which agrees with NNGSTR for
$N_{\nu}=3$ better than $1 \%$ when a coherent set of input parameters is used. In this approach, we can allow the parameter $N_{\nu}$ to vary while keeping the total width fixed.

Following eq. (1) the cross-section $\sigma_{0}(s)$ is written as

$\sigma_{0}(s)=\frac{12 \pi}{M_{\mathrm{Z}}^{2}} \frac{s \Gamma_{\mathrm{e}} N_{\nu} \Gamma_{\nu \bar{\nu}}}{\left(s-M_{\mathrm{Z}}^{2}\right)^{2}+s^{2} \Gamma_{\mathrm{Z}}^{2} / M_{\mathrm{Z}}^{2}}+\mathrm{W}$ terms,

where $\Gamma_{\nu \bar{\nu}}$ is the decay width of the $Z^{0}$ in a neutrino pair with standard model couplings, and $M_{\mathrm{Z}}, \Gamma_{\mathrm{Z}}$, and $\Gamma_{\mathrm{e}}$ are our measured values [4], for the $\mathrm{Z}^{0}$ mass, the total width and the electron partial width respectively. The results of the fit gives $N_{\nu}=3.14 \pm 0.24$ (stat.).

The systematic errors in our analysis come from the determination of the trigger efficiency, which gives an uncertainty of $\Delta N_{\nu}= \pm 0.04$, from the luminosity measurement, $\Delta N_{\nu}= \pm 0.03$, the determination of the selection efficiency, $\Delta N_{\nu}= \pm 0.02$, the background substraction, $\Delta N_{\nu}= \pm 0.09$, and the cosmic ray contamination, $\Delta N_{\nu}= \pm 0.02$. Moreover from the errors on our measurements of the $Z^{0}$ parameters $M_{\mathrm{Z}}, \Gamma_{\mathrm{Z}}$, and $\Gamma_{\mathrm{e}}$, from the top mass variation and the theoretical uncertainty on the parametrisation in eq. (2) we estimate a contribution to the systematic error of $\Delta N_{\nu}= \pm 0.05$. Adding all these systematic errors in quadrature, our final result is

$N_{\nu}=3.14 \pm 0.24$ (stat.) \pm 0.12 (syst.).

This corresponds to a $Z^{0}$ invisible width of

$\Gamma_{\mathrm{inv}}=524 \pm 40$ (stat.) \pm 20 (syst.) MeV. 


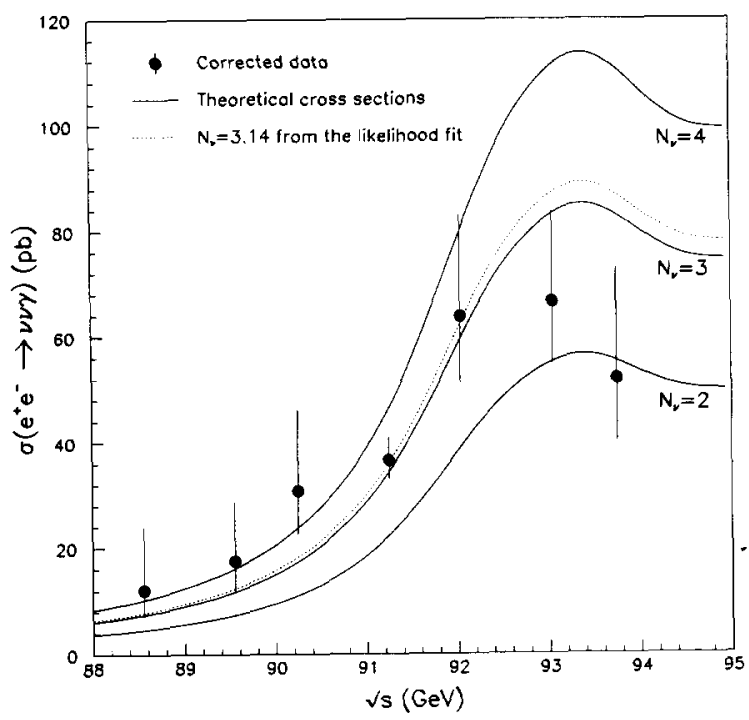

Fig. 4. Corrected single photon cross-sections $\left(45^{\circ}<\right.$ $\theta_{\gamma}<135^{\circ}$ and $E_{\gamma}>0.9 \mathrm{GeV}$ ) as a function of the CM energy, compared with the predictions of ref. [16].

This improves our previous published result [9]. The corrected cross-section is shown in fig. 4 as a function of the $\mathrm{CM}$ energy along with the expectations from $N_{\nu}=2,3,4$ and from our best fit.

\section{Conclusions}

We performed a direct determination of the number of light neutrino families by measuring the single photon cross-section at seven energies around the $Z^{0}$ resonance. With an integrated luminosity of $9.6 \mathrm{pb}^{-1}$, we observed 202 events with photon energies between 0.9 and $3.5 \mathrm{GeV}$ with an expected background of 29.9 events. From a maximum likelihood fit, we de- termine the number of light neutrinos with standard model coupling to be $N_{\nu}=3.14 \pm 0.24 \pm 0.12$. The corresponding value for the invisible decay width of the $Z^{0}$ into any weakly interacting particle is $\Gamma_{\mathrm{inv}}=$ $524 \pm 40 \pm 20 \mathrm{MeV}$.

\section{References}

[1] L. Trentadue et al., Z physics at LEP I, eds. G.Altarelli et al., CERN Yellow Book (1989), Vol. 1, CERN 8908 , p. 129.

[2] CDF Collab., F. Abe et al., Phys. Rev. Lett. 68 (1992) 447.

[3] C. Jarlskog, Phys. Lett. B 241 (1990) 579.

[4] L3 Collab., B. Adeva et al., Z. Phys. C 51 (1991) 179.

[5] A.D. Dolgov, L.B. Okun and V.I. Zakharov, Nucl. Phys. B 41 (1972) 197;

E. Ma and J. Okada, Phys. Rev. Lett. 41 (1978) 287; K.J. Gaemers, R. Gastmans and F.M. Renard, Phys. Rev. D 19 (1979) 1605;

G. Barbiellini, B. Richter and J. Siegrist, Phys. Lett. B 106 (1981) 414 .

[6] M. Chen, C. Dionisi, M. Martinez and X. Tata, Phys. Rep. 159 (1988) 201.

[7] The LEP Collabs., ALEPH, DELPHI, L3 and OPAL, Phys. Lett. B 276 (1992) 247.

[8] OPAL Collab., M.Z. Akrawy et al., Z. Phys. C 50 (1991) 373.

[9] L3 Collab., B. Adeva et al., Phys. Lett. B 275 (1992) 209.

[10] L3 Collab., B. Adeva et al., Nucl. Instrum. Methods A 289 (1990) 35.

[11] R. Brun et al., GEANT3 Users Guide, CERN/DD/EE/84.1.

[12] H. Fesefeldt, RWTH Aachen report PITHA 85/02 (1985).

[13] R. Bizzarri et al., Nucl. Instrum. Methods A 317 (1992) 463.

[14] D. Karlen, Nucl. Phys. B 289 (1987) 23.

[15] R. Miquel, C. Mana and M. Martinez, Z. Phys. C 48 (1990) 309;

F.A. Berends et al., Nucl. Phys. B 301 (1988) 583.

[16] O. Nicrosini and L. Trentadue, Nucl. Phys. B 318 (1989) 1 . 